NBER WORKING PAPER SERIES

\title{
COLLECTIVE INVESTMENT DECISION MAKING WITH HETEROGENEOUS TIME PREFERENCES
}

\author{
Christian Gollier \\ Richard Zeckhauser
}

Working Paper 9629

http://www.nber.org/papers/w9629

\author{
NATIONAL BUREAU OF ECONOMIC RESEARCH \\ 1050 Massachusetts Avenue \\ Cambridge, MA 02138 \\ April 2003
}

The views expressed herein are those of the authors and not necessarily those of the National Bureau of Economic Research.

(C)2003 by Christian Gollier and Richard Zeckhauser. All rights reserved. Short sections of text not to exceed two paragraphs, may be quoted without explicit permission provided that full credit including @notice, is given to the source. 
Collective Investment Decision Making with Heterogeneous Time Preferences

Christian Gollier and Richard Zeckhauser

NBER Working Paper No. 9629

April 2003

JEL No. H43, E43, E21, E40

\section{ABSTRACT}

We examine the investment decision problem of a group whose members have heterogeneous time preferences. In particular, they have different discount factors for utility, possibly not exponential. We characterize the properties of efficient allocations of resources and of shadow prices that would decentralize such allocations. We show in particular that the term structure of interest rates is decreasing when all members have DARA preferences. Heterogeneous groups should not use exponential discounting for their collective investment decisions even if all agents discount exponentially. We also exhibit conditions that lead the representative agent to have a rate of impatience that decreases with GDP per capita.

Christian Gollier University of Toulouse golllier@cict.fr
Richard Zeckhauser

John F. Kennedy School of Government Harvard University

79 JFK Street

Cambridge, MA 02138

and NBER

richard_zeckhauser@harvard.edu 


\section{Introduction}

Saving and investment decisions are among the most important choices of economic agents. They strongly affect the lifetime welfares of individuals and the prosperity of nations. Such decisions reflect time preferences. Most people prefer an immediate utility reward to the same reward experienced later. This pure preference for the present, or impatience, has long been recognized by economists and psychologists. The classical model introduced by Samuelson (1937) takes this into account by assuming that consumers maximize the discounted value of the flow of their utility, utilizing an exponentially decreasing discount factor, i.e., with a constant rate of impatience. However, the intensity of impatience is a subject that is little understood and intensely debated.

Frederick, Loewenstein and O'Donoghue (2002) survey several attempts to estimate individuals' discount rates. Two noteworthy findings emerge. First, high discount rates predominate. For example, Warner and Pleeter (2001) study actual financial decisions made by U.S. military servicemen, and find an estimated mean discount rate of $17.5 \%$ per year. Second, and more important, there is a spectacular variation across studies and within studies across individuals, with no convergence toward an agreed-upon or unique rate of impatience. In their study, Warner and Pleeter (2001) found that individual discount rates vary between $0 \%$ and $70 \%$ per year. Barsky, Juster, Kimball and Shapiro (1997) estimated a negative mean rate of impatience by using survey responses in the Health and Retirement Study. In the literature more generally, rates range from $-6 \%$ to $55700 \%$.

These variations could stem in part from differences in the time horizon considered in the various experiments and field studies. There is no reason to believe that consumers use the same discount rate per period when discounting utility over different time horizons. Strotz (1956) was the first economist to discuss horizon-dependent discount rates. Empirical evidence suggests that agents discount future happiness at a rate that declines with the time at which the happiness will be experienced. Most typically, people use "hyperbolic discounting", i.e., declining discount rate with respect to time-horizon, rather than exponential discounting. This leads to a time inconsistency problem that emerged recently as a "hot" topic in our profes- 
sion. ${ }^{1}$

This paper follows another path to explain the wide range of estimates for individual discount rates. There is no reason to believe that different consumers have identical time preferences for utility streams. Let us assume that sizeable disparities in discount rates arise because individuals strongly differ in their attitude towards time. Day-to-day evidence, say in pursuing education or bad habits, is compatible with heterogeneous time preferences. Such heterogeneity raises several questions that we explore in this paper.

When individuals use different rates of impatience to discount their future utility, it is not clear a priori which discount rate should be used, for example, for public investments. This raises the more general question of the aggregation of preferences in a group. We consider a general model where each agent maximizes a time additive lifetime utility. The discount rate is heterogenous in the population and it may depend upon either the time of receipt (hyperbolic discounting) or on current consumption. Agents may also have different instantaneous utility functions. We assume that the group is able to allocate consumption within the group in a Pareto-efficient way. We first show that the behaviour of the group towards time can be duplicated by a representative agent whose lifetime utility functional is also time additive. Time additivity is essential to define a concept of impatience. Rubinstein (1974) also examined the question of aggregating heterogenous rates of impatience, but he derives a solution only for exponential and logarithmic utility function in a two-period model.

One of the key findings of this paper is that if individuals have heterogeneous constant rates of impatience, the representative agent will not in general use a constant rate to discount the future. More precisely, we show that if individuals have decreasing absolute risk aversion (DARA), as would seem reasonable, then the representative agent has a declining discount rate. In short, heterogeneous individual exponential discounting yields collective hyperbolic discounting. Under some realistic calibrations of the economy, the collective discount factor duplicates either the one discussed by Loewenstein and Prelec (1992), or its simplified "quasi-hyperbolic" version (Laibson (1997)).

To get to this result, we need to examine how agents should share resources intertemporally in an exchange economy with no investment oppor-

\footnotetext{
${ }^{1}$ See for example Laibson (1997), Carrillo and Mariotti (2000), Benabou and Tirole (2000) and O'Donoghue and Rabin (2001).
} 
tunities, what might be labeled the multiple-cakes problem. Obviously, it is Pareto-efficient for the most impatient members to receive a larger share of the period's cake early in life; that share will be decreasing with time. This allocation is a best compromise between individual relative impatience and the agents' willingness to smooth consumption over time. This trait of individual preferences is measured by the concavity of their utility function. As shown by Wilson (1968), it is best to use the notion of (absolute) tolerance to consumption fluctuations over time. If $u($.$) denotes the utility function of$ an agent, her tolerance to fluctuations is measured by $T()=.-u^{\prime}(.) / u^{\prime \prime}($.$) . It$ is Pareto-efficient to request that more tolerant agents bear a larger share of fluctuations of aggregate incomes. Extending a well-known result by Wilson, we show that the group's tolerance to fluctuations in the per-capita income is the unweighted mean of its members' tolerance.

Turning to pure time preferences, we show that the rate of impatience of the representative agent equals a weighted mean of individual rates of impatience. These weights are proportional to the individual tolerances to consumption fluctuations. Intuitively, agents with a very low tolerance want to smooth their consumption independent of their degree of impatience. The group will therefore not take account of these agents when determining their collective degree of impatience. Except for exponential utility functions, the weights in computing the weighted mean of individual discount rates will fluctuate over time. When $T$ increases with wealth (DARA), those members with a smaller discount rate will see their weight increasing in tandem with time in parallel to their level of consumption. Therefore, we obtain that the rate of impatience of the representative agent decreases with time .

The fact that the representative agent uses hyperbolic discount factors in no way implies that the group faces a time consistency problem. Suppose that each individual in the group discounts future utility in an exponential way. As is well-known, such individuals will not want to modify their portfolio of future credit/saving contracts as time moves forward. In short, the allocation of future consumption will still be Pareto-efficient tomorrow. This future allocation will correspond to another set of Pareto weights. A time inconsistency problem arises only if some members in the group are themselves time inconsistent.

In the classical model of intertemporal choices, it is assumed that the rate of impatience is independent of wealth. We show by contrast that, even if individual rates of impatience are independent of consumption, the rate of impatience that should be used by the social planner is generally not indepen- 
dent of the group's per capita consumption. We provide a sufficient condition that guarantees that more developed economies should use a smaller rate of impatience.

As might be expected, the efficient allocations of resources over time can be acheived through a price mechanism. Thus we examine the characteristics of competitive prices that decentralize such Pareto-efficient allocations of resources. For the intertemporal choices, they are given by the term structure of interest rates. Cox, Ingersoll and Ross $(1985 \mathrm{a}, \mathrm{b})$ were the first to examine this question using a consumption-based approach. Whether we should use a decreasing rate to discount cash-flows occurring in the far distant future received intense debate. Significant long-term risks, such as global warming, are a new ingredient in the discussion, and beyond the scope of this analysis. In a risk free economy with no growth, the competitive interest rate equals the rate of impatience of the representative agent. Thus, our results sustain the recommendation to use a decreasing rate of interest to discount cash-flows occurring in a more distant future. The theoretical basis for this recommendation strongly differs from those developed earlier by Weitzman (2001) and Gollier (2002a,b).

\section{Assumptions on individual preferences}

We consider an economy or a group of heterogeneous agents indexed by $\theta$ in a type set $\Theta .^{2}$ Types are distributed according to cumulative distribution function $H: \Theta \rightarrow[0,1]$. We assume that the lifetime utility $\mathcal{U}(\theta)$ of consumer $\theta$ is time-additive. ${ }^{3}$ This excludes habit formation and anticipatory feelings. The lifetime utility of agent $\theta$ is evaluated at date 0 by

$$
\mathcal{U}(\theta)=\int_{0}^{\infty} u(c(t), t, \theta) d t
$$

\footnotetext{
${ }^{2}$ The duration of an agent's lifetime may be finite or infinite. If it is finite, $u^{\prime}$ is uniformly equal to zero after dead.

${ }^{3}$ Following Koopmans (1960), time additivity can be derived from the independence axiom stating that if two intertemporal prospects share a common outcome at a given date, then preference between them is determined solely by the remaining outcomes that differ.
} 
where $c($.$) is the consumption plan of the agent, and u(c, t, \theta)$ is the discounted felicity extracted by agent of type $\theta$ consuming $c$ at time $t$. We assume that

$$
u^{\prime}(c, t, \theta)=\frac{\partial u}{\partial c}(c, t, \theta)
$$

is continuously differentiable in $(c, t)$, and is nonincreasing in $c$. If $u^{\prime}$ is decreasing in $t$, consumers are impatient, i.e., at any given consumption level they value future marginal utility less than current marginal utility. We hereafter redefine the two well-known indexes of sensitivity of marginal utility either with respect to $t$ and with respect to $c$.

The instantaneous rate of pure time preference of agent $\theta$ consuming $c$ at time $t$ equals

$$
\delta(c, t, \theta)=-\frac{\partial \ln u^{\prime}(c, t, \theta)}{\partial t}=-\frac{\frac{\partial u^{\prime}}{\partial t}(c, t, \theta)}{u^{\prime}(c, t, \theta)} .
$$

It measures the rate at which marginal utility decreases with time with consumption held constant. This definition can be rewritten as

$$
u^{\prime}\left(c, t^{\prime}, \theta\right)=u^{\prime}(c, t, \theta) \exp \left[-\int_{t}^{t^{\prime}} \delta(c, \tau, \theta) d \tau\right] .
$$

Impatient people have a positive $\delta$. In the special case of a multiplicatively separable felicity function $u(c, t, \theta)=\beta(t, \theta) u(c, \theta), \delta$ is independent of $c$. If we add the assumption of exponential discounting $(\beta(t, \theta)=\exp (-\delta(\theta) t)), \delta$ is also independent of $t$. In the case of hyperbolic discounting, $\delta$ is independent of $c$, but is decreasing in $t$. In the face of the consistency problem that a non constant $\delta$ raises, we assume at this stage that agents can commit on their future consumption plan at date $t=0$.

In a parallel way, one can define the absolute aversion to consumption fluctuations over time through the following equation:

$$
A(c, t, \theta)=-\frac{\partial \ln u^{\prime}(c, t, \theta)}{\partial c}=-\frac{u^{\prime \prime}(c, t, \theta)}{u^{\prime}(c, t, \theta)} .
$$

Thus $A$ measures the rate at which marginal utility decreases with consumption at a given time. In the risk context, it corresponds to the Arrow-Pratt index of the concavity of $u$ with respect to $c$. As stated by Pratt (1964), in a static framework it satisfies:

$$
u^{\prime}\left(c^{\prime}, t, \theta\right)=u^{\prime}(c, t, \theta) \exp \left[-\int_{c}^{c^{\prime}} A(y, t, \theta) d y\right] .
$$


Under our assumptions, $A$ is nonnegative. Again, under the condition of a multiplicatively separable felicity function, $A$ would be independent of $t$. If we assume as well exponential felicity $(u(c, \theta)=-\exp (-a(\theta) c)), A$ would also be independent of $c$. But it is usually assumed that $A$ is decreasing in $c$, i.e., DARA applies.

$A$ and $\delta$ are two psychological traits of consumers that are essential to understand their saving and investment behavior. Suppose that agent $\theta$ has the opportunity to invest in various projects indexed by $j \in J$. Project $j$ yields a per-capita flow of incomes $\zeta_{j}():. R^{+} \rightarrow R$. The decision problem of the agent $\theta$ is to select the project that maximizes her lifetime utility:

$$
\max _{j \in J} \int_{0}^{\infty} u\left(c(t)+\zeta_{j}(t), t, \theta\right) d t
$$

Consider in particular a marginal investment project that costs $d x$ at time $t$ and that yields a benefit $e^{r d t} d x$ at time $t+d t .{ }^{4}$ Parameter $r$ is the return of this investment project. Assuming that the consumption path is continuously differentiable, the lifetime utility is unaffected by this investment if

$-u^{\prime}(c(t), t, \theta)+e^{r d t} u^{\prime}\left(c(t)+c^{\prime}(t) d t, t+d t, \theta\right)=u^{\prime}(c(t), t, \theta)\left[-1+e^{r d t} e^{-\delta d t} e^{-A c^{\prime}(t) d t}\right]$

is zero. This is equivalent to $r$ being equal to

$$
r(c, t, \theta)=\delta(c, t, \theta)+A(c, t, \theta) \frac{\partial c}{\partial t} .
$$

We say that $r(c, ., \theta)$ is the term structure of (forward) interest rates that sustains the consumption profile $c($.) of agent $\theta$. If all agents are identical, $r$ characterizes the competitive forward interest rates, where $c(t)$ is consumption per capita at time $t$. Reversing the argument, we can write that

$$
\frac{\partial c}{\partial t}=T(c, t, \theta)[r-\delta(c, t, \theta)]
$$

where function $T$ denotes the absolute tolerance to consumption fluctuations, which is the inverse of the absolute aversion to these fluctuations: $T(c, t, \theta)=$ $[A(c, t, \theta)]^{-1}=-u^{\prime}(c, t, \theta) / u^{\prime \prime}(c, t, \theta)$. We see that if $r$ is the equilibrium risk

\footnotetext{
${ }^{4}$ Obviously, these flows occur over a period of time of measure 0 , with no effect on $\mathcal{U}$. In reality, costs and benefits are incurred during respectively period $[t, t+\varepsilon]$ and period $[t+d t, t+d t+\varepsilon]$.
} 
free rate, an agent consuming $c$ at time $t$ should have a locally increasing consumption profile if $\delta(c, t, \theta)$ is less than $r$, and vice versa. This is the Keynes-Ramsey rule extended to non-separable felcity functions.

Condition (5) usefully extends the well-known property of optimal consumption when preferences exhibit exponential discounting. It shows the relationship between the psychological discount factor $\delta$ and the financial discount rate $r$ appropriately employed to discount monetary cash flows. The shadow price of time, $\rho$, is the sum of two terms. The first one is $\delta$, the rate of impatience of the agent. The second term comes from the preference for consumption smoothing exprssed by the concavity of $u$ with respect to $c$. When large consumption growth is expected, a large interest rate is required to induce agents to save. Otherwise, they would want to borrow money today to smooth the expected increase in their future incomes. Function $A$ measures the intensity of preference for consumption smoothing. For a given growth rate of consumption, the larger is $A$, the larger the interest rate required to sustain this growth rate, i.e., to fight the propensity to consume immediately.

\section{Efficient cake sharing with heterogeneous preferences}

Characterizing the optimal investment decision of a group of heterogeneous agents requires understanding how the group will share the cash flows generated by any such investment, namely how it divides the multiple cakes that become available, one per period. Suppose that agents of type $\theta$ are endowed with a flow $z(., \theta): R^{+} \rightarrow R$ of the single consumption good. We assume that endowments are risk free. An allocation is characterized by a set of consumption profiles $C(.,):. R^{+} \times \Theta \rightarrow R$, where $c(t, \theta)$ is the consumption of agent $\theta$ at time $t$. Such an allocation is feasible if at each instant of time average consumption equals average income:

$$
E C(t, \tilde{\theta})=z(t)=_{d e f} E z(t, \widetilde{\theta}) \quad \forall t
$$

where $\operatorname{Ef}(\widetilde{\theta})=\int_{\Theta} f(\theta) d H(\theta)$ is the mean of $f(\widetilde{\theta})$ with respect to the type distribution $H$.

The only restriction that we impose on the group's sharing of the cakes is that it be Pareto-efficient. An allocation $C(.,$.$) is Pareto-efficient if it is$ 
feasible and there is no other feasible allocation that raises the lifetime utility of at least one type without reducing the lifetime utility of the other types. To any such efficient allocation, there exists a weight function $\lambda():. \Theta \rightarrow R^{+}$ such that it is the solution of the following social planner's program:

$$
S W F(\lambda)=\max _{C(., .)} \quad E\left[\lambda(\widetilde{\theta}) \int_{0}^{\infty} u(C(t, \widetilde{\theta}), t, \widetilde{\theta}) d t\right] \quad \text { s.t. } \quad(7) .
$$

In his classic analysis of the static syndicate problem, Wilson (1968) considered a decision problem that is similar to (8). He examined a decision under uncertainty for expected-utility (EU) maximizers with heterogeneous utility functions, but homogeneous beliefs. Except for this restriction, the additivity property made in the EU model under static uncertainty and in the time-additive model (1) under dynamic certainty makes these two problems equivalent. Wilson (1968) proved that the optimal collective decision policy is isomorphic to the optimal decision policy of a representative agent who also maximizes the expected value of a function of consumption per capita in the group. The EU functional of the representative agent is additively separable with respect to the states of nature. Wilson's result can be extended to a dynamic framework, Constantinides (1982) has shown. The existence of a representative agent with such simple aggregative properties has become a cornerstone of the theories of finance and macroeconomics. In Wilson's model, the probability weights that are used to measure individual expected utilities are the same for all individuals given that beliefs are homogeneous. ${ }^{5}$ The parallel assumption for intertemporal choices under certainty is that agents use the same discounting function to measure their lifetime utility.

The following Proposition shows that the existence of a representative agent with a time separable lifetime utility does not require any restriction on time preference beyond the time separability of $\mathcal{U}(\theta)$ for all $\theta$.

Proposition 1 (Representative Agent) To any positive weight function $\lambda($.$) ,$ there exists a representative agent with a time-additive felicity function $v$ such that $S W F(\lambda)=\int_{0}^{\infty} v(z(t), t) d t$. Function $v$ is defined by

$$
v(z, t)=\max _{c(z, t, .)} E[\lambda(\widetilde{\theta}) u(c(z, t, \widetilde{\theta}), t, \widetilde{\theta})] \quad \text { s.t. } \quad \operatorname{Ec}(z, t, \widetilde{\theta})=z .
$$

The associated efficient allocation is characterized by $C(t, \theta)=c(z(t), t, \theta)$ for all $t$ and $\theta$.

\footnotetext{
${ }^{5}$ Leland (1980) reconsidered Wilson's model when agents have heterogeneous beliefs.
} 
Proof: This is a direct consequence of the additivity of $S W F(\lambda)$ with respect to types and time, which implies that

$$
E\left[\lambda(\widetilde{\theta}) \int_{0}^{\infty} u(C(t, \widetilde{\theta}), t, \widetilde{\theta}) d t\right]=\int_{0}^{\infty} E[\lambda(\widetilde{\theta}) u(C(t, \widetilde{\theta}), t, \widetilde{\theta})] d t . \mathbf{n}
$$

By this Proposition, we decompose the multiperiod maximization program (8) into a sequence of static maximization programs (9). By imposing at each time $t$ the feasible allocation of cake $z(t)$ that maximizes the weighted sum of instananeous felicities, the social planner obtains an ex ante allocation plan that maximizes the weighted sum of the members' lifetime utilities. The time additivity of individual preference functionals is, of course, essential to get this result.

Proposition 1 disentangles the two impacts that time has on the efficient sharing of the cake and on the felicity of the representative agent. First, it has a direct effect coming from the dependence of individual members' felicities on time. Second, it plays a role because income per capita, $z(t)$, is a function of time. It is useful to separate these two effects by defining $C(t, \theta)=c(z(t), t, \theta)$. In the following, we examine the properties of functions $v(.,$.$) and c(., ., \theta)$.

Because of the concavity of $u$ with respect to its first argument, the solution to program (9) is unique. Its first-order condition is written as

$$
\lambda(\theta) u^{\prime}(c(z, t, \theta), t, \theta)=\psi(z, t),
$$

for all $(z, t)$ and $\theta$, where $\psi$ is the Lagrange multiplier of the feasibility constraint associated with time $t$ and average endowment $z$. By the envelope theorem, the marginal value of wealth at time $t$ is the Lagrange multiplier associated with time $t$. Thus we have that

$$
\frac{\partial v}{\partial z}(z, t)=\psi(z, t)
$$

for all $(z, t)$.

\section{The group's tolerance to aggregate fluctu- ations}

We now characterize the group's tolerance to aggregate fluctuations in earnings. To do so, we need to determine how these fluctuations will be allocated 
among the different agents in the group. Consider a marginal increase in the per capita income $z$. At time $t$ with average consumption $z$, agent $\theta^{\prime} s$ sensitivity of consumption to such an increase is given by $\frac{\partial c}{\partial z}(z, t, \theta)$. This is referred to the marginal propensity to consume (MPC). It tells us how fluctuations in $z$ get transferred to fluctuations in individual consumption levels. Given the feasibility constraint $E c(z, t, \widetilde{\theta})=z$, it must be that, for all $(z, t)$,

$$
E \frac{\partial c}{\partial z}(z, t, \widetilde{\theta})=1
$$

The fluctuation of average consumption must equal the fluctuation of average earnings in the group. The following Proposition characterizes the MPC.

Proposition 2 (Cake-Sharing) The marginal propensity to consume of agent $\theta$ at time $t$ when the average endowment is $z$, is proportional to this agent's tolerance to consumption fluctuations evaluated at $c(z, t, \theta)$ :

$$
\frac{\partial c}{\partial z}(z, t, \theta)=\frac{T(c(z, t, \theta), t, \theta)}{E T(c(z, t, \widetilde{\theta}), t, \widetilde{\theta})} .
$$

Proof: Fully differentiating first-order condition (10) with respect to $z$ yields

$$
\lambda(\theta) u^{\prime \prime}(c(z, t, \theta), t, \theta) \frac{\partial c}{\partial z}(z, t, \theta)=\frac{\partial \psi}{\partial z}(z, t) .
$$

Eliminating $\lambda(\theta)$ by using (10) again, we rewrite the above condition as

$$
\frac{\partial c}{\partial z}(z, t, \theta)=-\frac{\frac{\partial \psi}{\partial z}(z, t)}{\psi(z, t)} T(c(z, t, \theta), t, \theta) .
$$

Moreover, combining this result with condition (12) implies that

$$
-\frac{\frac{\partial \psi}{\partial z}(z, t)}{\psi(z, t)} \operatorname{ET}(c(z, t, \widetilde{\theta}), t, \widetilde{\theta})=1 .
$$

Eliminating the ratio in (14) and (15) yields the result.

The cake-sharing Proposition states that more tolerant agents have larger marginal propensities to consume. It is intuitively appealing that people who are more tolerant to consumption fluctuations should be allocated a larger share of aggregate fluctuations. By contrast, agents who strongly dislike fluctuations, i.e., those with a small $T$, enjoy an efficient consumption plan 
that is relatively insensitive to aggregate fluctuations. Proposition 2 also shows that all agents have a nonnegative marginal propensity to consume out of aggregate incomes. All consumption levels are procyclical, but some are more procyclical than others.

Knowing how the group allocates fluctuations in aggregate earnings to different individuals determines the group's attitude towards fluctuations in the size of the cake. The group's tolerance to fluctuations in average earnings is given by

$$
T_{v}(z, t)={ }_{d e f}-\frac{\frac{\partial v}{\partial z}(z, t)}{\frac{\partial^{2} v}{\partial z^{2}}(z, t)} .
$$

When the per capita endowment $z($.$) is increasing in t$, an increase in $T_{v}$ will increase the group's propensity to invest in a normal project, i.e., a project that yields an increasing cash-flow over time. Equation (15) yields the following result.

Proposition 3 (Tolerance to Consumption Fluctuations) The group's absolute tolerance to consumption fluctuations is the mean of its members' tolerances:

$$
T_{v}(z, t)=E T(\widetilde{c}, t, \widetilde{\theta}) .
$$

The group's tolerance to fluctuations of per capita earnings equals the mean of its members' tolerances to consumption fluctuations. This observation has several consequences. For example, because this equation implies that

$$
\frac{\partial T_{v}}{\partial z}(z, t)=E\left[T^{\prime}(\widetilde{c}, t, \widetilde{\theta}) \frac{\partial c}{\partial z}(z, t, \widetilde{\theta})\right]=\frac{E\left[T^{\prime}(\widetilde{c}, t, \widetilde{\theta}) T(\widetilde{c}, t, \widetilde{\theta})\right]}{E T(\widetilde{c}, t, \widetilde{\theta})}
$$

we conclude that DARA is inherited by $v$ from $u$. In other words, if all members have a tolerance that increases in $c$, then the group has a tolerance that increases with $z$. This extends a result obtained by Carroll and Kimball (1996) to the group context. Notice that $v$ being DARA implies that the group as a whole is ready to pay more to smooth consumption when it is poor than when it is wealthy. 


\section{The group's rate of impatience and the yield curve}

In the classic case with homogenous exponential discount factors, individuals' consumption levels vary only with fluctuations in the aggregate endowment $z($.$) . When discount rates are heterogenous, time enters as an additional$ factor. We examine the partial derivative of individual consumption levels with respect to time: $\partial c / \partial t$. When the average income $z$ remains constant over time, it is intuitive that impatient people will trade later consumption for earlier consumption with those who are more patient. The impatient ones will have a decreasing consumption path, and vice versa.

Again, given the feasibility constraint, it must be the case that

$$
E \frac{\partial c}{\partial t}(z, t, \widetilde{\theta})=0
$$

When the average income remains constant over time, increases in consumption by some members of the group must be compensated by equivalent reductions in consumption by others. Fully differentiating the first-order condition (10) yields

$$
\lambda(\theta) \frac{\partial u^{\prime}}{\partial t}(c, t, \theta)+\lambda(\theta) u^{\prime \prime}(c, t, \theta) \frac{\partial c}{\partial t}=\frac{\partial \psi}{\partial t} .
$$

Using (2), (3) and (10) again to eliminate the Lagrange multiplier $\lambda$, we can rewrite the above equality as

$$
-\delta(c, t, \theta)-A(c, t, \theta) \frac{\partial c}{\partial t}=\frac{\frac{\partial \psi}{\partial t}}{\psi},
$$

or equivalently,

$$
\frac{\partial c}{\partial t}=-T(c, t, \theta)\left[\frac{\frac{\partial \psi}{\partial t}}{\psi}+\delta(c, t, \theta)\right] .
$$

Replacing $\partial c / \partial t$ in (18) by its expression from (19) yields

$$
\frac{\frac{\partial \psi}{\partial t}}{\psi}=-\frac{E[\delta(\widetilde{c}, t, \widetilde{\theta}) T(\widetilde{c}, t, \widetilde{\theta})]}{E[T(\widetilde{c}, t, \widetilde{\theta})]},
$$


where $\widetilde{c}=c(z, t, \widetilde{\theta})$. The next proposition characterizes the time profile of individual consumption plans when people have heterogenous discount rates. It flows from properties (19) and (20).

Proposition 4 (Individual Consumption Path) The increase in consumption through time of agent $\theta$ is decreasing in the agent's discount rate $\delta(\theta)$ :

$$
\frac{\partial c}{\partial t}(z, t, \theta)=T(c(z, t, \theta), t, \theta)[r(z, t)-\delta(c(z, t, \theta), t, \theta)]
$$

with

$$
r(z, t)=\frac{E[\delta(\widetilde{c}, t, \widetilde{\theta}) T(\widetilde{c}, t, \widetilde{\theta})]}{E T(\widetilde{c}, t, \widetilde{\theta})} .
$$

This result determines when more patient people should substitute current consumption for future consumption. In technical terms, it requires that $\partial c / \partial t$ be increasing in $\delta(\theta)$ when agents have the same tolerance to consumption fluctuations. The following Corollary exhibits the weaker property of single-crossing.

Corollary 1 Suppose that agents have the same tolerance to consumption fluctuations: $\partial T(c, t, \theta) / \partial \theta \equiv 0$. Then, individual consumption profiles satisfy the single-crossing property: $\forall\left(\theta, \theta^{\prime}\right) \in \Theta^{2}: \delta(c, t, \theta)>\delta\left(c, t, \theta^{\prime}\right) \quad \forall(c, t)$ implies that

$$
c(z, t, \theta)=c\left(z, t, \theta^{\prime}\right) \Longrightarrow \frac{\partial c}{\partial t}(z, t, \theta) \leq \frac{\partial c}{\partial t}\left(z, t, \theta^{\prime}\right) .
$$

Proof: This is a direct consequence of equation (21)

Equation (21) is reminiscent of property (6) of individual consumption profiles when the competitive interest rate is $r(z, t)$. In fact, $r$ defined by equation (22) would define the price of time that would decentralize the Pareto-efficient allocation $c(., .,$.$) when there is no growth. A term structure$ of interest rates satisfying (22) would induce agents to select consumption profiles that are compatible with the absence of growth. This shadow price of time is a weighted mean of the individual discount rates. Notice that the consumption path of agent $\theta$ increases locally in $t$ if and only if her rate of impatience is smaller than the market-determined shadow price of time. More patient members postpone their consumption to the future in exchange 
for a positive return on their savings. Because both $\delta$ and $r$ are a function of $z$ and $t$, efficient consumption profiles need not to be monotone.

We can now turn to the central aim of this paper, which is to characterize the aggregation of individual discount rates. Impatience comes from the fact that, seen from $t=0$, the marginal value of an increase in consumption decreases with the time at which it takes place. One can make impatience more explicit in the definition of the group's preferences by defining the group's instantaneous rate of impatience as

$$
\delta_{v}(z, t)={ }_{d e f}-\frac{\partial \ln \frac{\partial v}{\partial z}(z, t)}{\partial t}=-\frac{\frac{\partial^{2} v}{\partial z \partial t}(z, t)}{\frac{\partial v}{\partial z}(z, t)}=-\frac{\frac{\partial \psi}{\partial t}(z, t)}{\psi(z, t)}
$$

Combining conditions (23) and (20) yields the following result.

Proposition 5 (Collective Impatience) The instantaneous rate of pure preference for the present of the representative agent defined by (23) is a weighted mean of individual members' instantaneous rates:

$$
\delta_{v}(z, t)=\frac{E[\delta(\widetilde{c}, t, \widetilde{\theta}) T(\widetilde{c}, t, \widetilde{\theta})]}{E[T(\widetilde{c}, t, \widetilde{\theta})]} .
$$

Not surprisingly, the shadow price of time when there is no growth equals the psychological discount rate of the representative agent: $r(z, t)=\delta_{v}(z, t)$. In such an environment, a marginal investment incurring a cost at $t$ and yielding a benefit at $t+\Delta t$ would be socially desirable if and only if its net return would exceed $\delta_{v}(z(t), t)$. More generally, when there are aggregate fluctuations with earning profile $z(t)$, one can reinterpret equation (5) for the representative agent to determine that such an investment is socially desirable if its return exceeds

$$
\rho(t)=\delta_{v}(z(t), t)+\frac{z^{\prime}(t)}{T_{v}(z(t), t)} .
$$

Function $\rho($.$) characterizes the term structure of interest rates in such an$ economy. The important property (25) extends classical formulas from the literature on the term structure of interest rates $^{6}$ to allow for heterogenous

\footnotetext{
${ }^{6}$ See for example Cochrane (2001) for a recent evaluation. Earlier analyses can be found in Hansen and Singleton (1983), and Cox, Ingersoll and Ross (1985a, 1985b).
} 
rates of impatience among agents in the economy. As in (5), it decomposes the interest rate into its two components. The first one is the group's pure preference for the present $\delta_{v}$, whereas the second comes from the group's preference for consumption smoothing as measured by $T_{v}$. The next two sections focus on the first component. That is to say they examine the term structure of interest rates in a stationary state, when there is no economic growth.

\section{The term structure of the group's rate of impatience}

The group's rate of impatience can be rewritten as

$$
\delta_{v}(z, t)=\widehat{E}_{z, t} \delta(c(z, t, \widetilde{\theta}), t, \widetilde{\theta}),
$$

where $\widehat{E}_{z, t}$ is a "risk-neutral" expectation operator defined as

$$
\widehat{E}_{z, t} f(\widetilde{\theta})=\frac{\int f(\theta) T(c(z, t, \theta), t, \theta) d H(\theta)}{\int T(c(z, t, \theta), t, \theta) d H(\theta)} .
$$

The mean of individual rates of impatience is weighted by the corresponding individual tolerances to consumption fluctuations. This weighting of the mean of $\delta$ is intuitive. Patient agents will be willing to save strongly early in life only if they are sufficiently tolerant to the consumption fluctuations they will face. To illustrate, consider a group with two agents. Agent 1 has a high discount rate $\delta_{h}$ and is somewhat tolerant to consumption fluctuations. Agent 2, by contrast, has a lower discount rate $\delta_{l}$, but has a zero tolerance to consumption fluctuation. Despite his patience, agent 2 will prefer to smooth his consumption completely . Therefore, agent 1 will bear the entire burden of aggregate fluctuations. The group's attitude towards time is therefore determined entirely by agent 1's preferences. In particular, the group's degree of impatience will be the larger $\delta_{h}$.

As a direct consequence of the fact that $\delta_{v}$ is a weighted mean, it is bounded below and above by the smallest and largest individual rates of impatience:

$$
\min _{\theta \in \Theta} \delta(c(z, t, \theta), t, \theta) \leq \delta_{v}(z, t) \leq \max _{\theta \in \Theta} \delta(c(z, t, \theta), t, \theta)
$$


It is important to notice that the weighting function $T$ is a function of both $z$ and $t$. This is made explicit in the notation by indexing the expectation operator $\widehat{E}$ by $(z, t)$. Thus, even if $\delta$ is independent of $z$ and $t$, it is generally not true that $\delta_{v}$ is independent of these variables. We now examine the term structure of the group's rate of impatience.

Fully differentiating condition (24) with respect to $t$ and using condition (21) yields

$$
\begin{aligned}
\frac{\partial \delta_{v}}{\partial t}(z, t)= & 2\left(\widehat{E} \delta T^{\prime}\right)(\widehat{E} \delta)-\widehat{E} \delta^{2} T^{\prime}-(\widehat{E} \delta)^{2}\left(\widehat{E} T^{\prime}\right) \\
& +\widehat{E} \frac{\partial \delta}{\partial t}+\widehat{E} \frac{\partial \delta}{\partial c} T\left(\delta_{v}-\delta\right)-\widehat{E} \frac{1}{T} \frac{\partial T}{\partial t}\left(\delta_{v}-\delta\right)
\end{aligned}
$$

where $T, \delta$ and their derivatives are evaluated at $(c(z, t, \widetilde{\theta}), t, \widetilde{\theta})$. To simplify notation, we dropped the index to operator $\widehat{E}_{z, t}$. To examine this property, let us first focus on the traditional model of a group whose members are exponential discounters. In this benchmark case, individual felicity functions are multiplicatively separable: $u^{\prime}(c, t, \theta)=\beta(t, \theta) \nu^{\prime}(c, \theta)$. This means that $\delta$ is independent of $c$, and that $T$ is independent of $t$. The fact that individual members have exponential discount functions implies furthermore that $\beta(t, \theta)=\exp (-d(\theta) t)$, or that $\delta$ is also independent of $t$. This is a situation where the second line in property (27) vanishes.

The problem here is to determine whether the group as a whole should use exponential discounting when all of its members use exponential discounting. It trivially should when all members have the same discount rate. A more interesting case arises when individual preferences satisfy the ISHARA property. A felicity function has an Harmonic Absolute Risk Aversion (HARA) if its absolute risk tolerance is linear in consumption. Quadratic, exponential, power and logarithmic functions are HARA. A set of felicity functions satifies the Identically Sloped HARA (ISHARA) property if its members' absolute risk tolerances are linear in consumption with the same slope: $T^{\prime}(c, \theta)=1 / \gamma$. The set of felicity functions that satisfies this differential equation are as follows:

$$
u(c, t, \theta)=k \exp (-\delta(\theta) t)\left(\frac{c-a(\theta)}{\gamma}\right)^{1-\gamma} .
$$

These utility functions are defined over the consumption domain such that $\gamma^{-1}(c-a(\theta))>0$. When $\gamma>0$, parameter $a(\theta)$ is often referred to as the minimum level of subsistence. This preference set includes preferences with 
heterogeneous exponential utility functions $u(c, t, \theta)=-\beta(t, \theta) \exp (-A(\theta) c)$ when $\gamma$ tends to $+\infty$, and $a(\theta) / \gamma$ tends to $-1 / A(\theta)$. Taking $a(\theta)=0$ for all $\theta$, it also includes the set of power (and logarithmic) utility functions with the same relative concavity index $\gamma$ for all $\theta$. Under this set of conditions, equation (27) simplifies to

$$
\frac{\partial \delta_{v}}{\partial t}(z, t)=-T^{\prime}\left[\widehat{E} \delta^{2}-(\widehat{E} \delta)^{2}\right]
$$

Using Jensen's inequality, we conclude that the term structure of the social discount rate is decreasing if $T^{\prime}$ is a positive constant, and that it is increasing when $T^{\prime}$ is a negative constant. $T^{\prime}$ positive is a standard assumption in economics; it corresponds to decreasing absolute risk aversion (DARA) in the context of uncertainty. DARA means that agents have a tolerance to consumption fluctuations that increases with their wealth. In the next Proposition, we show that the constancy of $T^{\prime}$ can be relaxed at no additional cost.

Proposition 6 (Hyperbolic Collective Impatience) Suppose that agents have multiplicatively separable felicity functions with exponential discount: $u(c, t, \theta)$ $=k \nu(c, \theta) \exp (-\delta(\theta) t)$. The term structure of the social rate of impatience $\delta_{v}$ is decreasing (increasing) if all felicity functions $v(., \theta), \theta \in \Theta$, exhibit decreasing (increasing) absolute risk aversion.

Proof: We consider the case of DARA ( $T^{\prime}$ positive). Dividing equation (27) by $\widehat{E} T^{\prime}$, the discount rate $\delta_{v}$ is decreasing with respect to time if

$$
2(\widehat{E} \delta(\widetilde{\theta}))(\bar{E} \delta(\widetilde{\theta})) \leq(\widehat{E} \delta(\widetilde{\theta}))^{2}+\bar{E}(\delta(\widetilde{\theta}))^{2}
$$

where $\bar{E} \delta(\widetilde{\theta})=\int \delta(\theta) d F(\theta)$, and

$$
d F(\theta)=\frac{T(c(\theta), \theta) T^{\prime}(c(\theta), \theta)}{E T(c(\widetilde{\theta}), \widetilde{\theta}) T^{\prime}(c(\widetilde{\theta}), \widetilde{\theta})} d H(\theta) .
$$

Because $T^{\prime}$ is uniformly positive, $F$ can be interpreted as a cumulative probability function. Observe that

$$
2(\widehat{E} \delta(\widetilde{\theta}))(\bar{E} \delta(\widetilde{\theta})) \leq(\widehat{E} \delta(\widetilde{\theta}))^{2}+(\bar{E} \delta(\widetilde{\theta}))^{2}
$$


Moreover, we know that

$$
(\bar{E} \delta(\widetilde{\theta}))^{2} \leq \bar{E}(\delta(\widetilde{\theta}))^{2}
$$

Obviously, combining (30) and (31) yields (29), which concludes the proof

Notice that we don't restrict $T$ to be linear, nor assume any correlation between rates of impatience and degrees of tolerance to fluctuations. The monotonicity of these degrees of tolerance is the only thing that matters for the slope of the term structure of $\delta_{v}$. Simple intuition supports this important result. From equation (21), we know that more patient consumers have an increasing consumption profile. Under DARA, their tolerance to consumption fluctuation increases through time. This implies that when time goes forward, consumers with a low $\delta$ see their weight growing in the mean $\delta_{v}(z, t)=\widehat{E}_{z, t} \delta(\widetilde{\theta})$. This implies that the social rate of impatience decreases with time.

Example 1 Let us illustrate this result with a simple example. There are two groups of agents, respectively with constant rates of impatience $\delta_{l}$ and $\delta_{h}>\delta_{l}$. All agents have the same felicity function $\nu(c, \theta)=\min (b(c-a), d(c-a))$, with $0<d<b$. This function is piecewise linear with a kink at $c=a$. We consider the case where $b$ tends to infinity, which means that the left branch of the curve becomes vertical. Parameter a is the minimum level of subsistence. On the relevant domain $[a,+\infty$ [ of this limit function, agents have a nondecreasing tolerance (DARA), with a zero tolerance at $c=a$, and an infinite tolerance for all $c>a$. In this economy, any Pareto-efficient sharing of the cake produces a flip-flop consumption pattern. Prior to some identified date $\tau$, the patient group functions at subsistence, and the impatient group consumes any surplus. After time $\tau$, the impatient group falls to subsistence, and the patient group enjoys any surplus. As a consequence, the social rate of impatience $\delta_{v}(z, t)$ equals $\delta_{h}$ prior to $\tau$, and $\delta_{l}$ thereafter. The term structure is a simple downward step function in this case. Rates of impatience that are decreasing with time horizon are often refered to "hyperbolic" discounting. Phelps and Pollak (1968), then followed by Laibson (1997) and many others afterwards, introduced this stepwise functional form to describe observed psychological discount rates. This special case is often refered to the "beta-delta" model.

Example 2 This discounting functional would emerge as the socially efficient rule for less extreme examples. Let us just replace the piecewise linear 


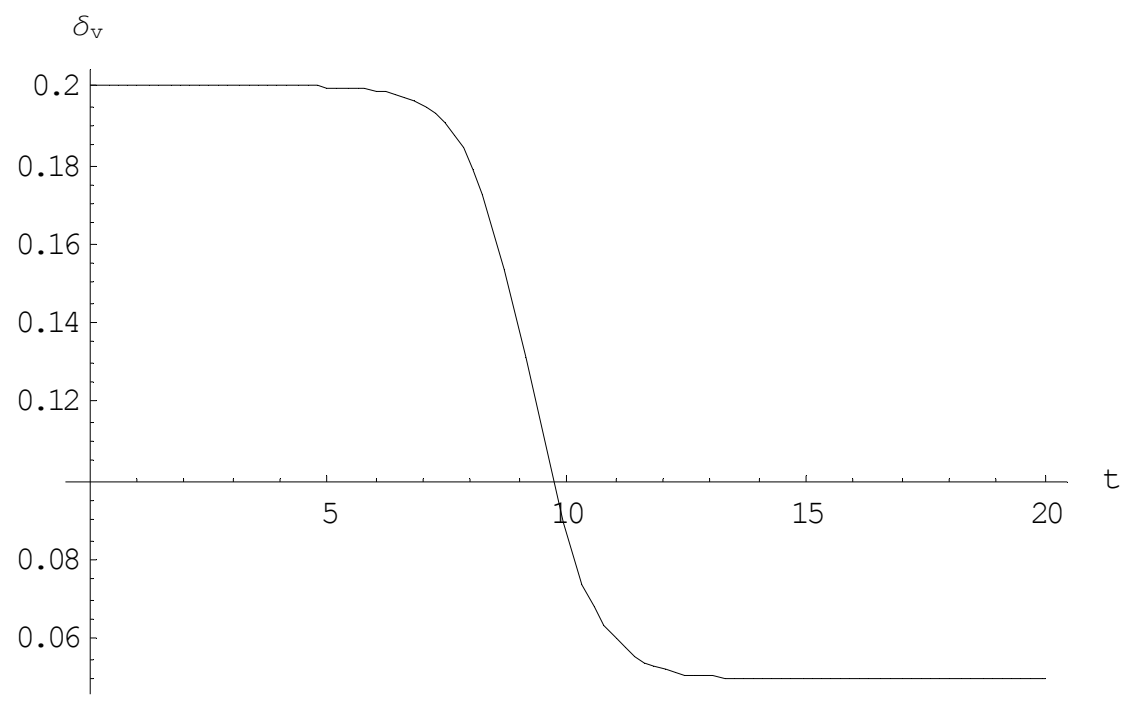

Figure 1: The discount rate as a function of time horizon for two-agent group with $\delta_{h}=20 \%$ and $\delta_{l}=5 \%$, when $\nu(c, \theta)=c^{0.9}$.

felicity function by a power felicity function. The two equally weighted groups have the same constant relative risk aversion $\gamma$. Under this "fair" efficient allocation of resources, the group's discount rate as a whole can be written as

$$
\delta_{v}(z, t)=\frac{\delta_{l}^{\frac{1+\gamma}{\gamma}} e^{-\frac{\delta_{l} t}{\gamma}}+\delta_{h}^{\frac{1+\gamma}{\gamma}} e^{-\frac{\delta_{h} t}{\gamma}}}{\delta_{l}^{\frac{1}{\gamma}} e^{-\frac{\delta_{l} t}{\gamma}}+\delta_{h}^{\frac{1}{\gamma}} e^{-\frac{\delta_{h} t}{\gamma}}} .
$$

When $\gamma$ tends to zero, this function of $t$ can be approximated by a downward step function with step levels at $\delta_{h}$ and $\delta_{l} \cdot{ }^{7}$ In Figure 1, we draw this function for $\delta_{h}=20 \%, \delta_{l}=5 \%$ and $\gamma=0.1$.

Example 3 Suppose that $\nu(c, \theta)=c^{1-\gamma} /(1-\gamma)$ and that $\delta(\theta)=\theta$. Suppose moreover that discount rates $\theta$ are distributed following a negative exponential law $\widetilde{\theta} \sim f(\theta)=e^{-\theta / \mu} / \mu$ on $\Theta \equiv[0,+\infty[$, with a mean $E \widetilde{\theta}=\mu$. We consider the Pareto-efficient allocation that corresponds to the weighting function $\lambda$

\footnotetext{
${ }^{7}$ The step occurs at time horizon $t=\left(\ln \delta_{h}-\ln \delta_{l}\right) /\left(\delta_{h}-\delta_{l}\right)$.
} 
such that $\lambda(\theta)=\theta^{\eta}$ for some scalar $\eta .^{8}$ In this illustration, it can be checked that

$$
\delta_{v}(z, t)=\frac{\eta+\gamma}{t+\frac{\gamma}{\mu}}
$$

which is independent of $z$. When relative risk aversion $\gamma$ tends to infinity, $\delta_{v}$ tends to $\mu$ uniformly for all $t$. When $\gamma$ tends to zero, $\bar{\delta}(z, t)$ tends uniformly to $\eta / t$. In Figure 2, we draw the maximum discount rate $\delta_{v}$ as a function of time when $\gamma=2$ (relative risk aversion) and $\mu=5 \%$ (mean discount rate). As seen in (32), the socially efficient discount rate $\delta_{v}$ declines with time $t$ as $1 /\left(t+\frac{\gamma}{\mu}\right)$. The discount factor $\beta(t)$ can be written as

$$
\beta(t)=\exp \left[-\int_{0}^{t} \delta_{v}(z, \tau) d \tau\right]=\left[1+\frac{\mu t}{\gamma}\right]^{-(\eta+\gamma)} .
$$

This is the functional form suggested by Loewenstein and Prelec (1992), who generalized earlier proposals made by Herrnstein (1981) and Mazur (1987).

It is useful to examine how consumption is allocated in this economy. The set of first-order conditions (10) combined with the feasibility constraints can be solved analytically to yield

$$
c(z, t, \delta)=\frac{\mu z}{\Gamma\left(\frac{\gamma+\eta}{\gamma}\right)}\left(\frac{t}{\gamma}+\frac{1}{\mu}\right)^{\frac{\gamma+\eta}{\gamma}} \delta^{\eta / \gamma} e^{-\frac{\delta t}{\gamma}},
$$

where $\Gamma(x)=\int_{0} \delta^{x-1} e^{-\delta} d \delta$ is the Gamma function. In Figure 3, we draw the efficient consumption plan for a few types when the average earnings in the population remain constant over time and are normalized to unity. We see again what drives the declining term structure of $\delta_{v}:$ At $t=0$, individual consumption levels and individual degrees of tolerance are positively related to the individual rates of impatience. This weighting leads to a social rate of impatience that is greater than $\mu$. As time goes forward, most resources go to those with low discount rates, and the social rate of impatience falls below $\mu$. Notice that, following condition (21) with $r \equiv \delta_{v}$, the consumption profile of agent $\theta$ is locally increasing as long as $\delta(\theta)$ is less than $\delta_{v}(z, t)$. Because $\delta_{v}$ is decreasing in $t$, consumption profiles of all agents with a rate of impatience $\delta(\theta)$

\footnotetext{
${ }^{8}$ One conception of fairness when all agents have the same utility function would set $\eta=1$. This implies that the mean weight of individuals' felicity over their (infinite) lifetime is the same for everyone: $\int_{0}^{\infty} \lambda(\theta) e^{-\theta t} d t=1$ for all $\theta$.
} 


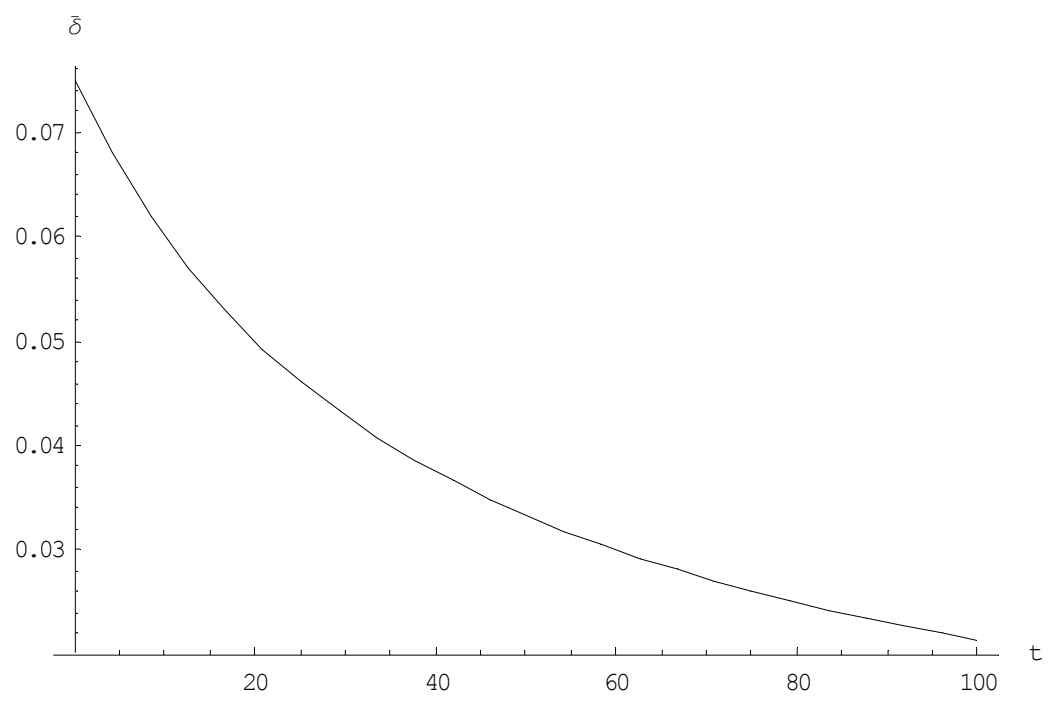

Figure 2: The social rate of impatience $\delta_{v}$ as a function of time.

less than $\delta_{v}(z, 0) \simeq 7.5 \%$ are hump-shaped, whereas those agents with a rate of impatience larger than $7.5 \%$ have decreasing consumption throughout. In general, efficient consumption profiles are either decreasing or hump-shaped under the assumptions of Proposition $6 .^{9}$

It follows immediately from equation (27) that one can relax the assumption that members of the group use exponential discounting. If $\partial \delta / \partial t$ is nonpositive for all $\theta \in \Theta$, that will just reinforce the negativity of the righthand side of this equation.

Corollary 2 Suppose that agents have multiplicatively separable felicity functions with hyperbolic discounting: $u(c, t, \theta)=k \exp (-\delta(t, \theta) t) \nu(c, \theta)$ and $\partial \delta / \partial t \leq$ 0 . The term structure of the social rate of impatience $\delta_{v}$ is decreasing if all felicity functions $v(., \theta)$ exhibit decreasing absolute risk aversion.

\footnotetext{
${ }^{9}$ The figure does not extend far enough to show the consumption for the individual with $\delta=1$ to begin to fall. The limit case is the agent with $\delta=0$; she is the only one to have a steadily increasing consumption plan.
} 


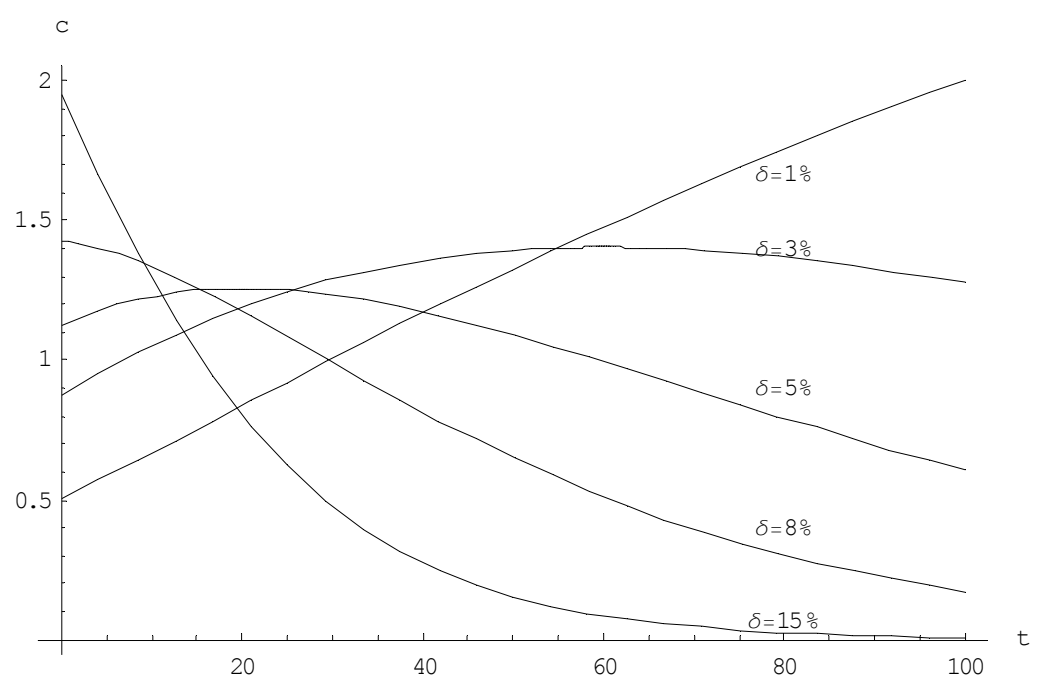

Figure 3: Efficient consumption path for agents with different discount rates $\delta$.

\section{A wealth effect on social impatience}

In the standard model of consumption, saving and growth, rates of impatience are assumed to be independent of consumption levels: $\partial \delta / \partial c \equiv 0$. However, it is often observed that wealthier economies are more patient. In our notation, this would mean that $\delta_{v}$ is decreasing in $z$. In this section, we examine whether these two assumptions can be compatible.

Observe that we found that $\delta_{v}$ is independent of $z$ in our three examples. This illustrates the following Proposition.

Proposition 7 If the members of the group have ISHARA preferences (28), the social rate of impatience is independent of the consumption per capita $z$.

Proof: Fully differentiating equation (24) with respect to $z$ and using property (13) yields

$$
(E T) \frac{\partial \delta_{v}}{\partial z}(z, t)=\widehat{E} \delta T^{\prime}-(\widehat{E} \delta)\left(\widehat{E} T^{\prime}\right)+\widehat{E} \frac{\partial \delta}{\partial c} T,
$$

where $T, \delta$ and their derivative are evaluated at $(c(z, t, \widetilde{\theta}), t, \widetilde{\theta})$, and where $\widehat{E}$ is the "risk-neutral" expectation operator defined by (26). For ISHARA 
preferences, $\delta$ is independent of $c$ and $T^{\prime}$ is a constant. It implies that the right-hand side of the above equality is zero. The driving force behind this result is that $\delta$ and $T^{\prime}$ are independent for ISHARA preferences.

Rubinstein (1974) obtained the same wealth irrelevancy property in the special case of exponential and logarithmic utility functions. In the following Proposition, by contrast, we assume that more patient people have a tolerance to fluctuations that is more sensitive to changes in consumption. In such a situation, the social rate of impatience will be decreasing with the consumption per capita in the economy, despite the fact that consumers' impatience does not depend on consumption.

Proposition 8 Suppose that the members of the group have a rate of impatience that is independent of their consumption: $\partial \delta / \partial c \equiv 0$. Suppose also that their tolerance to fluctuations is linear with respect to their consumption: $\partial T^{\prime} / \partial c \equiv 0$. The social rate of impatience at $t$ will be decreasing with the consumption level per capita if $\delta$ and $T^{\prime}$ evaluated at $t$ are anti-comonotone: $\forall\left(\theta, \theta^{\prime}\right) \in \Theta^{2}:\left[\delta(c, t, \theta)-\delta\left(c, t, \theta^{\prime}\right)\right]\left[T^{\prime}(c, t, \theta)-T^{\prime}\left(c, t, \theta^{\prime}\right)\right] \leq 0$.

Proof: Under these assumptions, we have that

$$
\widehat{E} \frac{\partial \delta}{\partial c} T=0
$$

and

$$
\widehat{E} \delta T^{\prime} \leq(\widehat{E} \delta)\left(\widehat{E} T^{\prime}\right) .
$$

From (35), it implies that $\delta_{v}$ is decreasing in $z$ at $t$.

A simple intuition supports this result. It comes from the fact that the social rate of impatience is a weighted mean of individual rates of impatience. When $\delta$ and $T^{\prime}$ are anti-comonotone, an increase in wealth differentially increases the weights associated with the lower rates of impatience. An increase in $z$ then pushes $\delta_{v}$ downwards.

In the special case of felicity functions exhibiting constant relative risk aversion (CRRA), viz. $u^{\prime}(c, t, \theta)=\beta(t, \theta) c^{-\gamma(\theta)}$, there is a negative relationship between relative risk aversion $\gamma(\theta)$ and $T^{\prime}(c, t, \theta)=1 / \gamma(\theta)$. Thus, the above Proposition applied to the case of CRRA felicity function implies that the rate of impatience is a decreasing function of societal wealth if more patient people are also less risk-averse. The following example illustrates this point. 


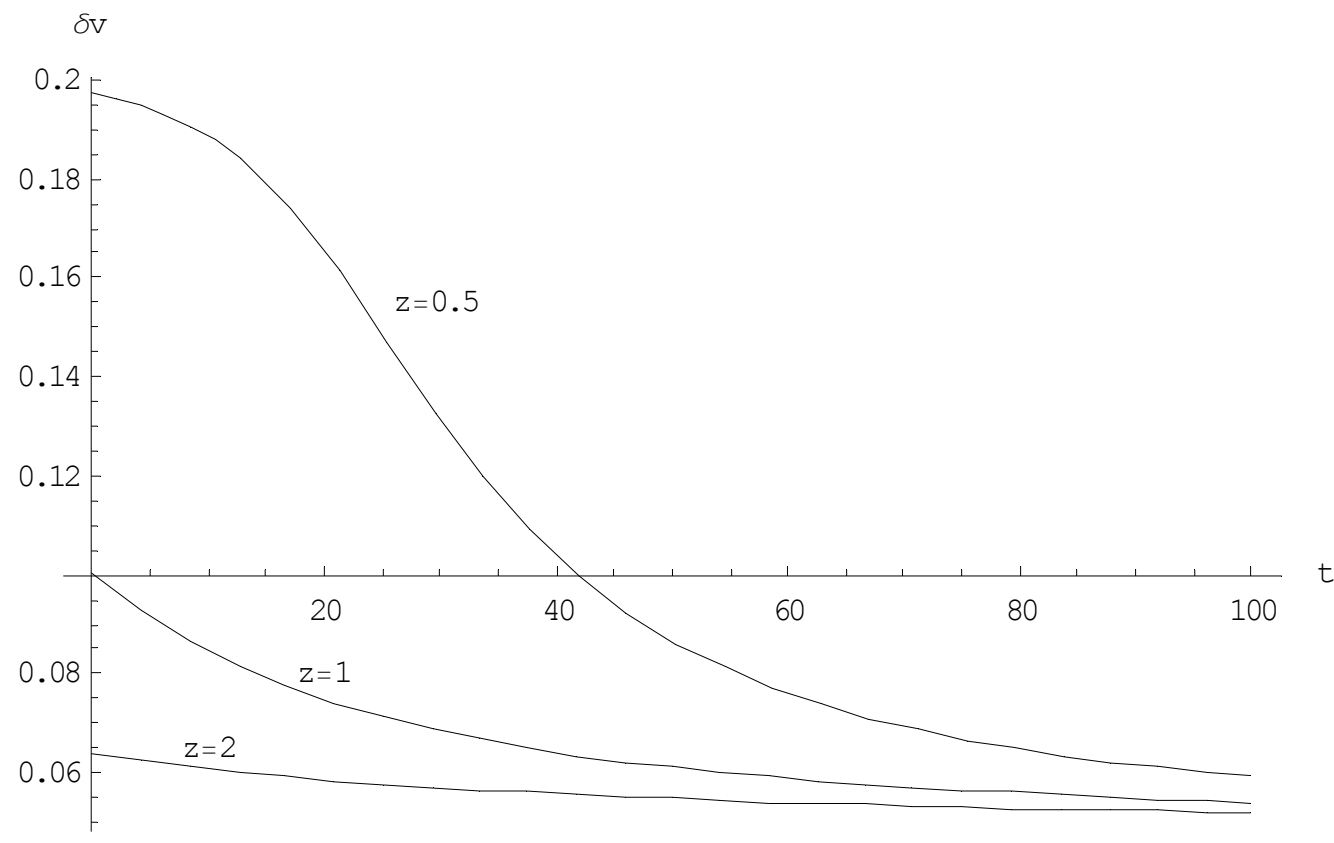

Figure 4: The term struture of the social rate of impatience when $u^{\prime}\left(c, t, \theta_{l}\right)=$ $e^{-0.05 t} c^{-1}$ and $u^{\prime}\left(c, t, \theta_{h}\right)=e^{-0.2 t} c^{-10}$.

Example 4 Consider an economy with two groups of equal size. Rates of impatience are constant, hence independent of time and consumption levels. The first group has low impatience rate $\delta_{l}$ and a logarithmic felicity function $\left(\gamma_{l}=1\right)$. The second group has a larger rate of impatience $\delta_{h}>\delta_{l}$ and a larger constant relative risk aversion $\gamma_{h}>1$. We derived numerically the Pareto-efficient allocation correponding to equal Pareto weights $\lambda_{l}=\lambda_{h}$, in the case of $\gamma_{h}=10, \delta_{l}=0.05$ and $\delta_{h}=0.20$. Figure 4 shows the term structure of the social rate of impatience when the consumption per capita $z$ equals $0.5,1$ and 2 . We see that a larger per capita consumption yields a smaller rate of impatience for all time horizons, as proved by Proposition 8.

\section{Interest rates and economic growth}

We now go back to the analysis of the term structure that sustains an efficient allocation of consumption. When there are no aggregate fluctuations, socially 
efficient interest rates just equal the social discount rates whose structure was examined in the previous two sections. When aggregate fluctuations prevail, the efficient interest rates are characterized by equation (25). When the economic growth rate $z^{\prime} / z$ is not zero, the second term in the right-hand side of (25) comes into play. In this section, we examine the properties of this consumption smoothing effect.

We first consider the direct effect of time on the consumption-smoothing term $z^{\prime} / T_{v}$. Because by definition we have that

$$
\frac{\partial T_{v}}{\partial t}(z, t)=\frac{\partial \delta_{v}}{\partial c}(z, t)
$$

for all $(z, t)$, the previous section gives us some information on this problem. Consider for example the case where agents have ISHARA preferences. Proposition 7 combined with property (36) tells us that $T_{v}$ is independent of $t$. It implies that the interest rate formula (37) is time invariant in this case. Consider alternatively the assumptions contained in Proposition 8, in particular the assumption that the discount rate $\delta$ and the derivative of absolute risk tolerance are negatively related. Using property (36), Proposition 8 states that, under these conditions, the absolute tolerance of the representative agent is decreasing with $t$. When the growth of the economy is positive, this is compatible with an upward sloping curve. Notice that this effect goes opposite to the impatience effect presented in Proposition 8.

We now turn to the role of growth on the consumption smoothing term of the interest rate. Suppose that the growth rate $z^{\prime} / z$ is constant over time. Observe that the asset pricing formula (25) can be rewritten as

$$
\rho(t)=\delta_{v}(z(t), t)+R_{v}(z(t), t) \frac{z^{\prime}(t)}{z(t)},
$$

where $R_{v}(z, t)=z / T_{v}(z, t)$ is the relative aversion to fluctuations of the representative agent. The benchmark case has agents with identical CRRA preferences. As is well-known, this implies that $R_{v}(z, t)$ is a constant. In the following proposition, we consider the alternative case where agents have heterogenous CRRA preferences. In a static context with uncertainty, Hara and Kuzmics (2002) show that this implies that the representative agent has an $R_{v}$ which is decreasing with respect to $z$. We now provide a shorter proof of this important result. As shown by Gollier (2002a), it implies that the yield curve is decreasing under the standard assumptions. 
Proposition 9 Suppose that agents have constant, identical rates of impatience, and that consumption per capita is growing at a constant rate. Then if agents have heterogenous but constant rates of relative risk aversion, the yield curve will be nonincreasing.

Proof: Because we assume that agents have homogenous rates of impatience, the first term in the right-hand side of (37) is independent of $t$. Moreover, we know that it also implies that $\partial R_{v} / \partial t$ vanishes. We are done if $\partial R_{v} / \partial z$ is nonpositive. Differentiating $R_{v}(z, t)=z / T_{v}(z, t)$ with respect to $z$ implies that this is the case if

$$
\operatorname{ET}(c(\widetilde{\theta}), t, \widetilde{\theta}) \leq z \frac{E T^{\prime}(c(\widetilde{\theta}), t, \widetilde{\theta}) T(c(\widetilde{\theta}), t, \widetilde{\theta})}{E T(c(\widetilde{\theta}), t, \widetilde{\theta})},
$$

or equivalently, since $T(c, t, \theta)=c / \gamma(\theta)$, if

$$
\left[E \frac{c(\widetilde{\theta})}{\gamma(\widetilde{\theta})}\right]^{2} \leq E c(\widetilde{\theta}) E \frac{c(\widetilde{\theta})}{(\gamma(\widetilde{\theta}))^{2}}
$$

By the Cauchy-Schwarz inequality, this is always true.

\section{Conclusion}

This paper shows that when agents have different rates of impatience, using a constant rate to discount cash-flow occurring at different dates - as is done in most cost-benefit analyses - is both inappropriate and inefficient. More specifically, under the widely accepted assumption of decreasing absolute risk aversion, employing a constant collective discount rate would tilt too much toward short- as opposed to long-term investments. The recognition of the existence of important differences in rates of impatience in the population should induce public decision makers to focus more on the long-term costs and benefits of public actions. The effect of heterogeneous time preferences should be taken seriously. In a simple calibration exercise in which we assumed that individual discount rates are distributed in the population according to a negative exponential law with a mean of $5 \%$ per year, we showed that the socially efficient discount rate should be around $7.5 \%$ per year for the short term, but that it should converge to $0 \%$ for long term cash-flows. 
Using the first number would accord with government practice. Using the second would elevate eyebrows.

We also showed that when there is a positive correlation between rates of impatience and relative risk aversion in the population, wealthier economies will be more patient. 


\section{References}

Barsky, R.B., F.T. Juster, M.S. Kimball and M. Shapiro, (1997), Preference parameters and behavioral heterogeneity: An experimental approach in the health and retirement study, Quarterly Journal of Economics, 537-79.

Benabou, R., and J. Tirole, (2000), Self-confidence: Intertemporal strategies, Princeton University discussion paper 209.

Carrillo, J.D., and T. Mariotti, (2000), Strategic ignorance as a self-disciplining device, Review of Economic Studies, 67:3, 529-544.

Caroll, C.D. and M. Kimball, (1996), On the concavity of the consumption function, Econometrica, 64, 981-92.

Cass, D. and J. Stiglitz, (1970), The structure of investor preferences and asset returns, and separability in portfolio allocation, Journal of Economic Theory, 2, 122-60.

Cochrane, J., (2001), Asset Pricing, Princeton University Press.

Constantinides, G.M., (1982), Intertemporal asset pricing with heterogeneous consumers and without demand aggregation, Journal of Business, 55, 253-67.

Cox, J., J. Ingersoll and S. Ross, (1985a), A theory of the term structure of interest rates, Econometrica, 53, 385-403.

Cox, J., J. Ingersoll and S. Ross, (1985b), An intertemporal general equilibrium model of asset prices, Econometrica, 53, 363384 .

Frederick, S., G. Loewenstein and T. O'Donoghue, (2002), Time discounting and time preference: A critical review, Journal of Economic Literature, 40, 351-401.

Gollier, C., (2002a), Discounting an uncertain future, Journal of Public Economics, 85, 149-166.

Gollier, C., (2002b), Time horizon and the discount rate, Jouranl of Economic Theory, forthcoming.

Hansen, L. and K. Singleton, (1983), Stochastic consumption, risk aversion and the temporal behavior of assets returns, Journal of Political Economy, 91, 249-268. 
Hara, C., and C. Kuzmics, (2001), Representative consumer's risk aversion and efficient risk-sharing rules, mimeo, Cambridge University.

Herrnstein, R., (1981), Self-control as response strength, in Quantification of steady-state operant behavior, C.M. Bradshaw, E. Szabadi and C.F. Lowe Eds, Elsevier/North Holland.

Koopmans, T.C., (1960), Stationary ordinal utility and impatience, Econometrica 28, 287-309.

Laibson, D.I., (1997), Golden eggs and hyperbolic discounting, Quarterly Journal of Economics, 62, 443-479.

Leland, H.E., (1980), Who Should Buy Portfolio Insurance?, Journal of Finance, 35, 581-596.

Loewenstein, G., and D. Prelec, (1991), Negative time preference, American Economic Review, 81, 347-352.

Mazur, J.E., (1987), An adjustment procedure for studying delayed reinforcement, in The effect of delay and intervening events on reinforcement value, M.L. Commons, J.E. Mazur, J.A. Nevin and H. Rachlin Eds, Hillsdale, NJ: Erlbaum.

O'Donoghue, T., and M. Rabin, (2001), Choice and procrastination, Quarterly Journal of Economics, 116:1, 121-160.

Phelps, E.S., and R. Pollack, (1968), On second-best national saving and game equilibrium growth, Review of Economic Studies, 35, 185-199.

Samuelson, P.A., (1937), A note on the measurement of utility, Review of Economic Studies, 4, 155-161.

Strotz, R.H., (1956), Myopia and inconsistency in dynamic utility maximization, Review of Economic Studies, 23:3, 165-180.

Warner, J.T., and S. Pleeter, (2001), The personal discount rate: Evidence from military downsizing programs, American Economic Review, 95:4, 547-580.

Weitzman, M.L., (2001), Gamma discounting, American Economic Review, 91, 260-271.

Wilson, R. (1968). "The theory of syndicates", Econometrica 36, $113-132$. 